\section{(1) \\ CrossMark}

\title{
Diagnostic utility and accuracy of rapid on-site evaluation of bronchoscopic brushings
}

\author{
Daniel P. Steinfort ${ }^{1,2}$, Tracy L. Leong ${ }^{1}$, Irena F. Laska ${ }^{1}$, Anne Beaty ${ }^{3}$, Alpha Tsui ${ }^{3}$ \\ and Louis B. Irving ${ }^{1}$
}

Affiliations: ${ }^{1}$ Dept of Respiratory Medicine, Royal Melbourne Hospital, Parkville, VC, Australia. ${ }^{2}$ Dept of Medicine, University of Melbourne, Parkville, VC, Australia. ${ }^{3}$ Dept Pathology, Royal Melbourne Hospital, Parkville, VC, Australia.

Correspondence: Daniel P. Steinfort, Dept of Respiratory Medicine, Royal Melbourne Hospital, Grattan St, Parkville 3050, VC, Australia. E-mail: daniel.steinfortamh.org.au

ABSTRACT The aim of the study was to determine the accuracy of rapid on-site examinations, performed on transbronchial brushings of peripheral pulmonary lesions, in determining final bronchoscopic diagnosis. In addition to determining if rapid on-site examination impacts procedural outcomes.

A prospective cohort study of consecutive patients with peripheral pulmonary lesions, which had been located by radial endobronchial ultrasound, was undertaken. Bronchoscopy was terminated if rapid on-site examination demonstrated diagnostic malignant material. Non-diagnostic rapid on-site examination resulted in further bronchoscopic sampling, including transbronchial lung biopsy and/or sampling from different locations.

128 peripheral pulmonary lesions were located by endobronchial ultrasound in 118 patients. The final bronchoscopic diagnoses included nonsmall cell lung cancer $(n=76)$, carcinoid $(n=3)$, and metastatic malignancy $(n=3)$. Procedure times were significantly shorter for procedures when rapid on-site examinations demonstrated malignancy compared to those where rapid on-site examination was nondiagnostic ( $19 \pm 8$ min versus $31 \pm 11 \mathrm{~min}$, respectively; $\mathrm{p}<0.0001)$. In four procedures, initial negative rapid on-site examination results prompted redirection of sampling from alternate bronchial segments, resulting in positive diagnostic tissue being obtained. Positive and negative predictive value of rapid on-site examination for a malignant bronchoscopic diagnosis was $63(97 \%)$ out of 65 , and $43(68 \%)$ out of 63 , respectively.

Rapid on-site examination of brushing specimens has a very high, positive, predictive value for bronchoscopic diagnosis of cancer and shortens the bronchoscopy procedure times. It has the potential to reduce complications, improve cost-effectiveness, and may improve diagnostic performance via live feedback.

@ERSpublications

ROSE of bronchial brushings during bronchoscopy for PPL can shorten procedure times and increase diagnostic yield http://ow.ly/FASOW 


\section{Introduction}

Bronchoscopy is associated with high diagnostic accuracy in the assessment of malignant central airway lesions, although performance of routine bronchoscopy is known to be poor in assessment of peripheral pulmonary lesions (PPL) $[1,2]$. Various localisation techniques are used to improve diagnostic sensitivity, including fluoroscopy, radial probe endobronchial ultrasound (EBUS), and electromagnetic navigation. Despite imperfect diagnostic performance, bronchoscopy remains an appropriate initial investigation for PPL, due to the lower complication rate and procedural cost in comparison to percutaneous biopsy $[3,4]$.

Rapid on-site examination (ROSE) of cytology specimens is advocated by multiple experts as being integral to optimal performance of transbronchial needle aspiration (TBNA) [5, 6]. Reported advantages include the reduced number of biopsies performed, a lower procedural risk, and an improved cost-effectiveness $[7,8]$, although this finding is not universal $[9,10]$, Furthermore, a high degree of agreement between the on-site and final pathologic evaluation during EBUS-TBNA has been observed [11].

Use of ROSE during fluoroscopically guided bronchoscopic sampling of PPL has been suggested to improve diagnostic yield $[12,13]$. Use of ROSE to examine peripheral bronchial brushings, during performance of electromagnetic navigation bronchoscopy, has also been reported, although the contribution to procedural outcomes is unknown [14, 15].

Radial probe EBUS allows localisation of peripheral bronchogenic lesions with visualisation of the typical ultrasound appearance (fig. 1) confirming, to the proceduralist, that the lesion has been reached. Diagnostic sensitivity has been estimated by meta-analysis at $73 \%$ [16], and in selected patient groups may be non-inferior to that of percutaneous biopsy [3]. We postulated that the use of ROSE during radial EBUS bronchoscopy may allow an earlier termination of procedure, due to the confirmation that diagnostic tissue has been retrieved and, therefore, may improve diagnostic sensitivity by redirecting sampling to adjacent bronchial segments in the event of a negative ROSE finding. We prospectively assessed the impact of routine ROSE during the performance of radial EBUS bronchoscopy and present these findings in this report. We also sought to characterise the negative predictive value and false-positive rate, and to identify any pitfalls associated with its use.

\section{Materials and methods}

Institutional review board approval was granted for the performance of this study.

We prospectively recorded data from all patients undergoing investigation of PPL with radial EBUS in whom EBUS demonstrated successful localisation of the target lesion (fig. 1). Given the poor yield in patients in whom EBUS is unable to locate PPLs [17, 18], our institution does not perform targeted sampling if the lesion is not located, therefore, these patients were not included in the analysis.

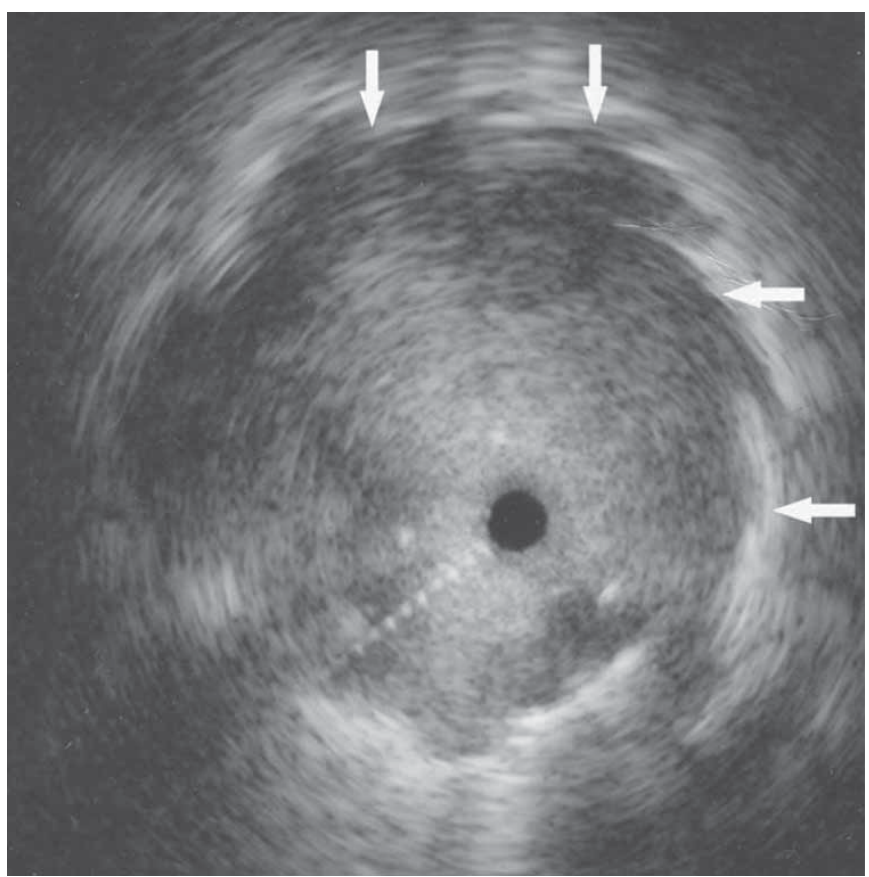

FIGURE 1 Radial, endobronchial ultrasound image with typical findings indicating peripheral mass lesion. The hyperechoic line (arrows) indicates the ultrasound interface between solid lesion and surrounding air-filled lung. 
Performance of radial EBUS

Bronchoscopy was performed, as previously described, under conscious sedation [3]. All bronchoscopies were performed by experienced proceduralists. Following topical anaesthesia, lignocaine $2 \%$, the $20 \mathrm{MHz}$ radial EBUS probe (UM-BS20-26R; Olympus, Tokyo, Japan) and guide sheath were introduced. Visible bronchial segments were sequentially examined, directed by computed tomography chest imaging, until the characteristic ultrasound signal indicating the presence of solid lesions was demonstrated. The EBUS probe was then removed and sampling instruments introduced through the guide sheath, with sampling performed under fluoroscopic vision.

\section{Performance of ROSE}

Bronchial brush specimens were smeared onto two, positively charged, frosted glass slides. One was rapidly air dried and stained with a rapid Romanowsky stain for ROSE (Quick Dip; POCD Scientific, Artarmon, Australia) (fig. 2a) and the other slide was fixed immediately in 95\% alcohol for Papanicolaou stain (Quick Dip; POCD Scientific) (fig. 2b). The rapid Romanowsky staining and the microscopic examination were performed within the bronchoscopy suite by a cytology scientist and the ROSE diagnosis was communicated immediately to the proceduralists.

Diagnostic ROSE specimens were characterised as those clearly demonstrating the typical cytological features of malignancy; namely nuclear size and shape variation between single cells and cells within a group. Non-diagnostic specimens were those where (rapid Romanowsky) ROSE failed to convincingly demonstrate these features, including where specimens demonstrated only the appearance of benign epithelial cells or where specimens demonstrated a paucity of malignant cells (e.g. $<10$ groups of $\geqslant 5$ cells).

\section{Outcome measures}

We sought to establish the diagnostic performance of ROSE compared with the final procedural diagnosis, which was the final, reported diagnosis following an examination of all the specimens obtained at bronchoscopy (brushings, transbronchial lung biopsy (TBLB), bronchial washings).

We also aimed to determine if any procedural findings influenced diagnostic performance of either the ROSE or final pathology reports. Procedural findings recorded included EBUS position (within PPL versus adjacent to PPL), EBUS appearance (solid versus patchy), procedural time, final procedural diagnosis that included specific results of brushings, TBLB, and bronchial washings.

\section{Statistical analysis}

Continuous variables were expressed as mean $\pm \mathrm{SD}$, with comparisons performed using t-test for normally distributed variables and nonparametric testing (Mann-Whitney) for non-normally distributed variables. Categorical variables were presented as summary statistics, including simple proportions, with comparisons performed using Chi-squared test. All reported confidence intervals are two-sided. Sensitivity, specificity, and accuracy of the two methods were calculated according to standard definition. For all

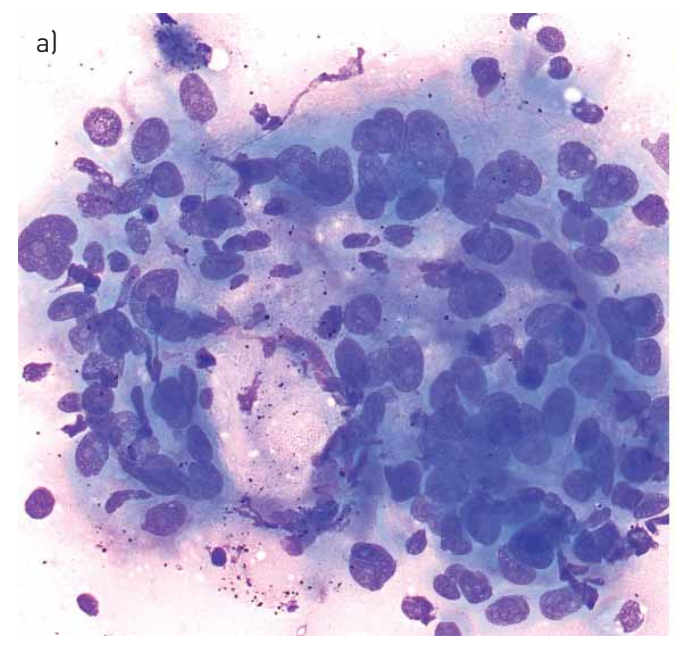

b)

FIGURE 2 Bronchial brushings specimen stained a) with the rapid Romanowsky stain, demonstrating malignant epithelial cells and b) by Papapnicolaou method, showing a hypercellular specimen with abundant malignant cells occurring singularly and in groups. 
analyses, the level of statistical significance was set at 0.05. Analyses were performed using Stata Statistical Software 10.0 (StataCorp, College Station, TX, USA).

\section{Results}

From August 2011 to April 2013, 128 PPLs in 118 patients were located bronchoscopically using radial EBUS. Characteristics of patients and PPLs are presented in table 1. No procedural complications occurred.

ROSE was positive for malignancy in 65 lesions (50.8\%), and the final procedural pathologic diagnosis was of malignancy in 83 lesions (65\%). In four patients, where initial ROSE was negative for malignancy, subsequent redirection of sampling resulted in positive ROSE specimens.

Final pathologic diagnosis differed from the ROSE diagnosis in 22 cases: in two PPL where ROSE identified malignancy, the final pathologic examination did not identify definite evidence of malignancy. In 20 cases, final pathologic examination of all retrieved specimens identified malignancy when ROSE had not identified malignant cells. Thus, when compared to the final procedural diagnosis, ROSE demonstrated sensitivity for bronchoscopic detection of malignancy of $76 \%$, a specificity of $96 \%$, a positive predictive value of $97 \%$ and negative predictive value of $68 \%$.

Mean \pm SD procedure time was significantly shorter for procedures where ROSE was positive for malignancy $(19.2 \pm 7.8 \mathrm{~min}$ versus $31.0 \pm 10.6 \mathrm{~min}, \mathrm{p}<0.0001)$. No association was observed between lesion size and ROSE outcome. A positive ROSE result was significantly more likely where the EBUS probe was located within the lesion (odds ratio $(\mathrm{OR}) 5.5$ versus adjacent, $\mathrm{p}<0.001)$ and where the EBUS image demonstrated a solid lesion (OR 14 versus patchy appearance, $\mathrm{p}=0.003$ ). The same associations were noted between the EBUS appearance and the final procedural diagnosis (within versus adjacent OR 5.6, $\mathrm{p}=0.0001$; solid versus patchy OR 6.3, $\mathrm{p}=0.0001)$.

TBLB was performed in 86 patients. TBLB was positive for malignancy in 37 patients and negative in 49 patients. Brushings diagnosis and TBLB diagnosis were concordant in 68 (79\%) patients. In 13 cases, brushings were positive with TBLB negative, and five patients had positive TBLB despite negative brushings diagnosis.

\section{Cases with false-positive ROSE}

The initial ROSE specimens (rapid Romanowsky stained) and Papanicolaou-stained brushing specimens in two patients where ROSE indicated a false-positive result were re-examined by an expert lung pathologist.

\section{TABLE 1 Participant demographics and clinical information}

$\begin{array}{ll}\text { Characteristics } & 118 \\ \text { Patients } & 68 \\ \text { Sex } & 50 \\ \text { Male } & 70(46-88) \\ \text { Female } & 2.8 \pm 1.4(0.8-10.0) \\ \text { Age years } & \\ \text { Lesion size cm } & 128 \\ \text { Final procedural diagnosis } & 44 \\ \text { Total } & 19 \\ \text { Adenocarcinoma } & 13 \\ \text { Squamous carcinoma } & 3 \\ \text { NSCLC, not otherwise specified } & 3 \\ \text { Carcinoid } & 46 \\ \text { Metastatic malignancy } & \\ \text { Benign } & 128 \\ \text { Lesion location } & \\ \text { Total } & \\ \text { Right lobe } & 44(34) \\ \text { Upper } & 9(7) \\ \text { Middle } & 19(15) \\ \text { Lower } & \\ \text { Left lobe } & 30(23) \\ \text { Upper } & 8(6) \\ \text { Lingula } & 18(14) \\ \text { Lower } & 19\end{array}$

Data are presented as $\mathrm{n}, \mathrm{n}$ (range), $\mathrm{n} \pm \mathrm{SD}$ (range) or $\mathrm{n}(\%)$. NSCLC: nonsmall cell lung cancer. 
They concurred with the initial cytology opinions, though noted in one case that the ROSE specimen demonstrated atypical cells suspicious for malignancy but not sufficient to confirm a diagnosis of non-small cell lung cancer (NSCLC) (fig. 3).

Interestingly, in both lesions where final procedural pathology diagnosis suggested a false-positive ROSE diagnosis, subsequent biopsy procedures confirmed a malignant diagnosis for PPL (one percutaneous biopsy and one surgical wedge resection).

\section{Discussion}

Clinical utility of ROSE is contingent upon close agreement with final procedural diagnosis. This has been confirmed in performance of TBNA and evidence suggests it is likely to improve diagnostic yield, shorten procedure time, reduce the number of biopsies performed, lower procedural risk, and improve cost-effectiveness $[7,8]$. Particularly important to our approach of terminating the procedure in the event of ROSE demonstrating findings of malignancy is that the technique has a high, positive predictive value with respect to the final procedural diagnosis. Our recorded positive predictive value of $97 \%$ is highly reassuring, and it is interesting to observe that both lesions with false-positive ROSE findings, as determined by the final bronchoscopic diagnosis, were ultimately confirmed to be malignant by further investigations. High, positive predictive values allow for intra-procedural decision to be made on the basis of ROSE findings with confidence, consistent with subsequent final pathologic diagnosis. It supports our approach of termination of procedure in the event of ROSE revealing malignancy, without further sampling.

Our findings also suggest the ability of ROSE to inform intra-procedural decision making contributes to shorter procedure times, as the reliable positive results preclude the need for further sampling, particularly TBLB. Shortening of procedure times can improve theatre/endoscopy efficiency, thereby reducing procedure costs, and will minimise physiologic perturbation known to affect patients undergoing bronchoscopy [19]. While this benefit may be greatest in patients with severe background lung disease, it is worth noting that the magnitude of change in carbon dioxide and $\mathrm{pH}$, following bronchoscopy, does not differ between patients with and without pulmonary disease [20]. Avoiding use of TBLB forceps will also reduce procedural costs through the reduced use of consumables, and through obviating the need for post-TBLB chest radiographs, which is routine at many institutions. Given most complications of bronchoscopy relate to performance of TBLB [21], the ability of ROSE to obviate this sampling method may also reduce the complication rate of bronchoscopy, as well as the costs associated with managing complications.

Few studies have reported the use of ROSE to examine bronchoscopic brushings [13-15], and to our knowledge only one study has examined the effect of its use on diagnostic yield [12]. UcHIDA et al. [12] noted an improvement was achieved as a result of redirection of sampling in the event of a non-diagnostic procedure. We observed a similar finding, although smaller in magnitude, with redirection of EBUS
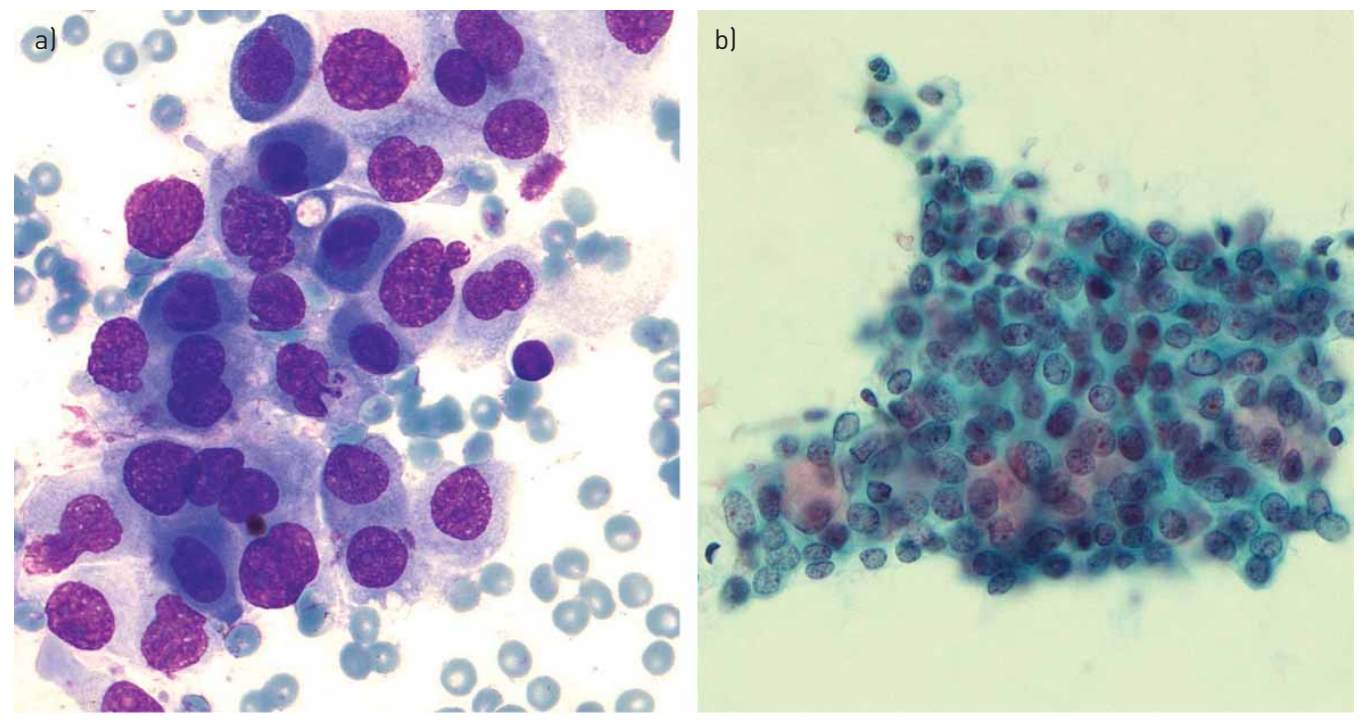

FIGURE 3 Bronchial brushing specimen stained a) by rapid Romanowsky stain, demonstrating cells with enlarged, mildly pleomorphic nuclei with slightly irregular nuclear contour, suspicious for malignancy; and b) by the Papanicolaou method, showing sheets of benign epithelial cells. Mildly atypical epithelial cells are present, although the overall appearances favour reactive changes. 
examination/sampling resulting in diagnostic specimens being retrieved in four cases. This is an improvement in diagnostic yield of $6 \%$ which, though small, is very important in the cases in which it was achieved. UCHIDA et al. [12] reported a positive predictive value of $100 \%$, and a lower false-negative rate (9.2\%) than we have. It is unclear if this represents differing utility of the staining method used (rapid Shorr stain), but our findings demonstrate the potential for false-positive results, and emphasise the need for individual institutions to audit their technique, should they commence ROSE for brushing specimens with a view to guiding intra-procedural decision making.

Our findings also suggest, as has been advised regarding the use of ROSE for EBUS-TBNA [11], that negative or indeterminate results should be interpreted with caution. Multiple studies of bronchoscopic accuracy in assessment have noted varying diagnostic performance of the sampling modalities routinely undertaken [22]. Most reports are consistent with our observation that brushings are associated with the highest diagnostic yield, but that in a minority of cases TBLB may be the sole technique to retrieve diagnostic specimens [22]. For this reason, and given the above observation regarding the potential improvement in diagnostic yield from redirection of sampling, we suggest that negative ROSE should not dissuade proceduralists from performing TBLB and that subsequent EBUS examination of adjacent bronchial segments should be strongly considered.

The utility of individual sampling techniques in bronchoscopic investigation of suspected malignancy varies between reports, with studies variably identifying TBLB [23], or brushings [22, 24] as being associated with the highest yield. What is universally noted is that maximal yield is achieved by a combination of techniques [22-25]. Our results confirm this, with brushings being associated with a higher yield where TBLB was performed, but for five lesions TBLB was the sole sampling identifying malignancy. This represents $6 \%$ of all cases where TBLB was performed, which we feel is an important increment in diagnostic yield.

One important lesson regarding ROSE for brushing specimens is the challenge in assessment of the cellular material, both in terms of the stain used and the cellular features of material being examined. When utilising ROSE during TBNA, the difference between normal (lymphocytes) and abnormal (epithelial malignancy) cells is clear. In contrast, differentiating between normal or reactive pneumocytes and NSCLC cells may sometimes be challenging. The rapid Romanowsky stain is not routinely used to assess bronchial brushing specimens and does not show the definite nuclear chromatin, nucleoli and cytoplasmic staining that the Papanicolaou stain shows. However, it does show an air drying artefact, whereby the stained material appears larger than that seen in a Papanicolaou stain, which can be useful in assessing nuclear size and shape variation seen in well differentiated carcinomas. Also, acellular material, such as mucin, is demonstrated more readily with the rapid Romanowsky stain.

Bronchial brushing smears are often markedly hypercellular with normal bronchial material presenting as single cells and thick cellular groups, often admixed with mucus or cellular debris. This can make the detection of scanty malignant cells and well differentiated malignancy difficult, especially in the setting of a rapid assessment on unfamiliar stained material.

Proposals for determining adequacy of on-site specimens following TBNA have been suggested, which characterise adequacy based on the volume and purity of lymphocytes seen per field [26, 27]. It is difficult to apply such criteria to peripheral bronchial brushings, as the distinction between normal and abnormal is so clear cut as for lymph node aspirates; only subtle morphologic differences may be observed between reactive cells with nuclear atypia versus malignant, or between normal bronchial epithelial cells and low grade tumours with only mild pleomorphism. The assessment is further limited at ROSE as nuclear chromatin and cytoplasmic morphology is difficult to assess with the rapid Romanowsky stain.

\section{Limitations}

Our study is a prospective observational (non-randomised) cohort of consecutive patients. Diagnostic performance of EBUS bronchoscopy is consistent with that suggested by previous meta-analyses [16] and, therefore, we feel our findings have good internal and external validity. Nevertheless, a randomised multicentre study would ideally be required to confirm the ability of ROSE on brushings specimens, in order to improve procedural diagnostic yield/sensitivity, complication rates and confirm cost benefit.

We used the rapid Romanowsky staining, whereas other studies, investigating the use of ROSE on brushings, used a modified Gill-Shorr method [28]. Different staining methods used in the preparation of lymph node TBNA specimens are associated with varying sensitivity [29-31], suggesting the important need to examine the optimal staining method for ROSE on brushings in future studies.

We have not examined factors that may allow for differentiation between potential false-negative results and true-negative results. Prior studies of radial EBUS have shown that diagnostic yields are poorer when the probe is positioned adjacent to the PPL [17, 18, 25, 32, 33], and that specific EBUS image 
characteristics are suggestive of benign aetiology [34, 35]. It is possible that future bronchoscopy decision making algorithms might incorporate such findings to suggest whether further sampling (e.g. with TBLB or TBNA) or redirected sampling would be of more utility in investigation of PPL. Such questions should be addressed in future studies. Factors influencing EBUS visualisation success have also been studied and should be incorporated into management algorithms.

We have not reported the feasibility of molecular testing in the event of diagnostic brushings precluding performance of TBLB. Histology specimens have generally been used for the performance of epidermal growth factor receptor or anaplastic lymphoma kinase testing, though the utility of cytology specimens obtained by EBUS-guided TBNA for molecular diagnosis is well established [5]. Pulmonary lesions diagnosed by radial EBUS differ from those diagnosed by TBNA, as they are likely to be early stage, in which case subsequent surgical resection will afford a specimen more than sufficient for molecular testing. For patients who do not undergo surgical resection (or staging EBUS-TBNA) following radial EBUS diagnostic of NSCLC, our laboratory is still able to routinely perform molecular testing on cytology specimens derived from bronchial brushings specimens (data not shown) using techniques previously reported [36, 37]. Therefore, we do not believe patient care is compromised by avoiding TBLB, and given the potential for improved procedure duration, cost and safety, we suggest diagnostic ROSE of bronchial brushings specimens should preclude TBLB.

We have not examined the cost-effectiveness for the routine use of ROSE. The main cost relating to ROSE is of cytologist/cytology scientist time and will vary between institutions. We believe ROSE is likely to be cost-effective, given the shortened procedure time (reducing theatre usage and staff time) and given the small, but significant, number of cases where ROSE potentially ensured a diagnostic procedure, thereby obviating further invasive diagnostic investigations.

\section{Conclusions}

Rapid on-site cytological evaluation of bronchial brushings specimens, with assessment by cytology scientists, may be performed with very high, positive predictive values, which allow for intra-procedural decision making to be made on the basis of ROSE findings with confidence. When used to guide intra-procedural decision making, use of ROSE can shorten procedure times and increase diagnostic yield of bronchoscopic investigation of PPL. ROSE of bronchial brushings specimens has the potential to reduce complications and be cost-beneficial.

Negative ROSE results should be interpreted with caution, with performance of TBLB recommended in such cases and the examination of adjacent bronchial segments should also be considered. Further study is required to determine the optimal staining method and to develop diagnostic/adequacy criteria for on-site examination of brushings specimens.

\section{References}

1 Wallace JM, Deutsch AL. Flexible fiberoptic bronchoscopy and percutaneous needle lung aspiration for evaluating the solitary pulmonary nodule. Chest 1982; 81: 665-671.

2 Roth K, Hardie JA, Andreassen AH, et al. Predictors of diagnostic yield in bronchoscopy: a retrospective cohort study comparing different combinations of sampling techniques. BMC Pulm Med 2008; 8: 2.

3 Steinfort DP, Vincent J, Heinze S, et al. Comparative effectiveness of radial probe endobronchial ultrasound versus CT-guided needle biopsy for evaluation of peripheral pulmonary lesions: a randomized pragmatic trial. Respir Med 2011; 105: 1704-1711.

4 Steinfort DP, Liew D, Irving LB. Radial probe EBUS versus CT-guided needle biopsy for evaluation of peripheral pulmonary lesions: an economic analysis. Eur Respir J 2013; 41: 539-547.

5 Nakajima T, Yasufuku K. How I do it-optimal methodology for multidirectional analysis of endobronchial ultrasound-guided transbronchial needle aspiration samples. J Thorac Oncol 2011; 6: 203-206.

6 Yasufuku K, Fleury Feith J. Cytological specimens obtained by endobronchial ultrasound-guided transbronchial needle aspiration: sample handling and role of rapid on-site evaluation. Ann Pathol 2012; 32: e35-e46.

7 Baram D, Garcia RB, Richman PS. Impact of rapid on-site cytologic evaluation during transbronchial needle aspiration. Chest 2005; 128: 869-875.

8 Trisolini R, Cancellieri A, Tinelli C, et al. Rapid on-site evaluation of transbronchial aspirates in the diagnosis of hilar and mediastinal adenopathy: a randomized trial. Chest 2011; 139: 395-401.

9 Griffin AC, Schwartz LE, Baloch ZW. Utility of on-site evaluation of endobronchial ultrasound-guided transbronchial needle aspiration specimens. Cytojournal 2011; 8: 20.

10 Yarmus L, Van der Kloot T, Lechtzin N, et al. A randomized prospective trial of the utility of rapid on-site evaluation of transbronchial needle aspirate specimens. J Bronchology Interv Pulmonol 2011; 18: 121-127.

11 Nakajima T, Yasufuku K, Saegusa F, et al. Rapid on-site cytologic evaluation during endobronchial ultrasound-guided transbronchial needle aspiration for nodal staging in patients with lung cancer. Ann Thorac Surg 2013; 95: 1695-1699.

12 Uchida J, Imamura F, Takenaka A, et al. Improved diagnostic efficacy by rapid cytology test in fluoroscopy-guided bronchoscopy. J Thorac Oncol 2006; 1: 314-318. 
Bandoh S, Fujita J, Tojo Y, et al. Diagnostic accuracy and safety of flexible bronchoscopy with multiplanar reconstruction images and ultrafast Papanicolaou stain: evaluating solitary pulmonary nodules. Chest 2003; 124: 1985-1992.

14 Pearlstein DP, Quinn CC, Burtis CC, et al. Electromagnetic navigation bronchoscopy performed by thoracic surgeons: one center's early success. Ann Thorac Surg 2012; 93: 944-949.

15 Lamprecht B, Porsch P, Pirich C, et al. Electromagnetic navigation bronchoscopy in combination with PET-CT and rapid on-site cytopathologic examination for diagnosis of peripheral lung lesions. Lung 2009; 187: 55-59.

16 Steinfort DP, Khor YH, Manser RL, et al. Radial probe endobronchial ultrasound for the diagnosis of peripheral lung cancer: systematic review and meta-analysis. Eur Respir J 2011; 37: 902-910.

17 Tay JH, Irving L, Antippa P, et al. Radial probe endobronchial ultrasound: factors influencing visualization yield of peripheral pulmonary lesions. Respirology 2013; 18: 185-190.

18 Huang CT, Ho CC, Tsai YJ, et al. Factors influencing visibility and diagnostic yield of transbronchial biopsy using endobronchial ultrasound in peripheral pulmonary lesions. Respirology 2009; 14: 859-864.

19 Wallbridge PD, Hannan LM, Joosten SA, et al. Clinical utility of sequential venous blood gas measurement in the assessment of ventilatory status during physiological stress. Intern Med J 2013; 43: 1075-1080.

20 Chhajed PN, Rajasekaran R, Kaegi B, et al. Measurement of combined oximetry and cutaneous capnography during flexible bronchoscopy. Eur Respir J 2006; 28: 386-390.

21 Pue CA, Pacht ER. Complications of fiberoptic bronchoscopy at a university hospital. Chest 1995; 107: 430-432.

22 Griffin JP, Zaman MK, Niell HB, et al. Diagnosis of lung cancer: a bronchoscopist's perspective. J Bronchology Interv Pulmonol 2012; 19: 12-18.

23 Mak VH, Johnston ID, Hetzel MR, et al. Value of washings and brushings at fibreoptic bronchoscopy in the diagnosis of lung cancer. Thorax 1990; 45: 373-376.

24 Schreiber G, McCrory DC. Performance characteristics of different modalities for diagnosis of suspected lung cancer: summary of published evidence. Chest 2003; 123: 115S-128S.

25 Kuo CH, Lin SM, Lee KY, et al. Endobronchial ultrasound-guided transbronchial biopsy and brushing: a comparative evaluation for the diagnosis of peripheral pulmonary lesions. Eur J Cardiothorac Surg 2014; 45: 894-898.

26 Nayak A, Sugrue C, Koenig S, et al. Endobronchial ultrasound-guided transbronchial needle aspirate (EBUS-TBNA): a proposal for on-site adequacy criteria. Diagn Cytopathol 2012; 40: 128-137.

27 Alsharif M, Andrade RS, Groth SS, et al. Endobronchial ultrasound-guided transbronchial fine-needle aspiration: the University of Minnesota experience, with emphasis on usefulness, adequacy assessment, and diagnostic difficulties. Am J Clin Pathol 2008; 130: 434-443.

28 Omiya H, Nagatomo I, Yamamoto S, et al. Rapid staining with the modified Gill-Shorr method for reliable, rapid bronchoscopic diagnosis. Acta Cytol 2006; 50: 444-446.

29 Louw M, Brundyn K, Schubert PT, et al. Comparison of the quality of smears in transbronchial fine-needle aspirates using two staining methods for rapid on-site evaluation. Diagn Cytopathol 2012; 40: 777-781.

30 Diacon AH, Koegelenberg CF, Schubert P, et al. Rapid on-site evaluation of transbronchial aspirates: randomised comparison of two methods. Eur Respir J 2010; 35: 1216-1220.

31 Diacon AH, Schuurmans MM, Theron J, et al. Transbronchial needle aspirates: comparison of two preparation methods. Chest 2005; 127: 2015-2018.

32 Kurimoto N, Miyazawa T, Okimasa S, et al. Endobronchial ultrasonography using a guide sheath increases the ability to diagnose peripheral pulmonary lesions endoscopically. Chest 2004; 126: 959-965.

33 Yamada N, Yamazaki K, Kurimoto N, et al. Factors related to diagnostic yield of transbronchial biopsy using endobronchial ultrasonography with a guide sheath in small peripheral pulmonary lesions. Chest 2007; 132: 603-608.

34 Kuo CH, Lin SM, Chen HC, et al. Diagnosis of peripheral lung cancer with three echoic features via endobronchial ultrasound. Chest 2007; 132: 922-929.

35 Chao TY, Lie $\mathrm{CH}$, Chung $\mathrm{YH}$, et al. Differentiating peripheral pulmonary lesions based on images of endobronchial ultrasonography. Chest 2006; 130: 1191-1197.

36 Tsai TH, Yang CY, Ho CC, et al. Multi-gene analyses from waste brushing specimens for patients with peripheral lung cancer receiving EBUS-assisted bronchoscopy. Lung cancer 2013; 82: 420-425.

37 Iwabu N, Izumo T, Nakamura Y, et al. Molecular analysis of liquid cytological samples collected by bronchoscopy with radial endobronchial ultrasonography and guide sheath. Ann Thorac Cardiovasc Surg 2014; 20: Suppl., 692-696. 